EPJ Web of Conferences 13, 02004 (2011)

DOI: $10.1051 /$ epjconf/20111302004

C Owned by the authors, published by EDP Sciences, 2011

\title{
Interplay between chiral and deconfinement phase transitions
}

\author{
Fukun $\mathrm{Xu}^{1}$, Tamal K. Mukherjee ${ }^{1,2}$, Huan $\mathrm{Chen}^{3}$, and Mei Huang ${ }^{1,2}$ \\ 1 Institute of High Energy Physics, Chinese Academy of Sciences, Beijing, China \\ 2 Theoretical Physics Center for Science Facilities, Chinese Academy of Sciences, Beijing, China \\ 3 Istituto Nazionale di Fisica Nucleare (INFN), Sezione di Catania, Italy
}

\begin{abstract}
By using the dressed Polyakov loop or dual chiral condensate as an equivalent order parameter of the deconfinement phase transition, we investigate the relation between the chiral and deconfinement phase transitions at finite temperature and density in the framework of three-flavor Nambu-Jona-Lasinio (NJL) model. It is found that in the chiral limit, the critical temperature for chiral phase transition coincides with that of the dressed Polyakov loop in the whole $(T, \mu)$ plane. In the case of explicit chiral symmetry breaking, it is found that the phase transitions are flavor dependent. For each flavor, the transition temperature for chiral restoration $T_{c}^{\chi}$ is smaller than that of the dressed Polyakov loop $T_{c}^{\mathcal{D}}$ in the low baryon density region where the transition is a crossover, and, the two critical temperatures coincide in the high baryon density region where the phase transition is of first order. Therefore, there are two critical end points, i.e, $T_{C E P}^{u, d}$ and $T_{C E P}^{s}$ at finite density. We also explain the feature of $T_{c}^{\chi}=T_{c}^{\mathcal{D}}$ in the case of 1st and 2nd order phase transitions, and $T_{c}^{\chi}<T_{c}^{\mathcal{D}}$ in the case of crossover, and expect this feature is general and can be extended to full QCD theory.
\end{abstract}

\section{Introduction}

The interplay between chiral symmetry breaking and confinement as well as the chiral and deconfinement phase transitions at finite temperature and density are of continuous interests [1-9]. The two transitions are characterized by the breaking and restoration of chiral and center symmetry, which are well defined in two extreme quark mass limits, respectively. In the chiral limit when the current quark mass is zero $m=0$, the chiral condensate is the order parameter for the chiral phase transition. When the current quark mass goes to infinity $m \rightarrow \infty$, QCD becomes pure gauge $S U(3)$ theory, which is center symmetric in the vacuum, and the usually used order parameter is the Polyakov loop [1].

The relation between the chiral and deconfinement phase transitions has attracted more interest recently in studying the phase diagram at high baryon density region [10]. It is conjectured in Ref. [11] that in large $N_{c}$ limit, a confined but chiral symmetric phase, which is called quarkyonic phase can exist in the high baryon density region. It is very interesting to study whether this quarkyonic phase can survive in real QCD phase diagram, and how it competes with nuclear matter and the color superconducting phase [12]. (However, it is worthy of noticing that in Ref. [13], it is found that at zero chemical potential, the lattice results for the thermodynamical properties have a very mild dependence on the number of colors.)

Lattice QCD at the current stage cannot go to very high baryon density. For zero chemical potential, previous lattice results show that the chiral and deconfinement phase transitions occur at the same temperature, e.g, in
Ref. [14-18], and also in review papers $[19,20]$. In recent years, three lattice groups, MILC group [21], RBCBielefeld group [22] and Wuppertal-Budapest group [2325] have studied the chiral and deconfinement phase transition temperatures with almost physical quark masses. The RBC-Bielefeld group claimed that the two critical temperatures for $N_{f}=2+1$ coincide at $T_{c}=192(7)(4) \mathrm{MeV}$. The Wuppetal-Budapest group found that for the case of $N_{f}=2+1$, there are three transition temperatures, the transition temperature for chiral restoration of $u, d$ quarks $T_{c}^{\chi(u d)}=151(3)(3) \mathrm{MeV}$, the transition temperature for chiral restoration of $s$ quark $T_{c}^{\chi(s)}=175(2)(4) \mathrm{MeV}$ and the deconfinement transition temperature $T_{c}^{d}=176(3)(4) \mathrm{MeV}$. From this result, we can read that the critical temperatures for different transitions are different. According to the Wuppetal-Budapest group, this is the consequence of the crossover nature.

In the framework of QCD effective models, there is still no dynamical model which can describe the chiral symmetry breaking and confinement simultaneously. The main difficulty of effective QCD model to include confinement mechanism lies in that it is difficult to calculate the Polyakov loop analytically. Currently, the popular models used to investigate the chiral and deconfinement phase transitions are the Polyakov Nambu-Jona-Lasinio model (PNJL) [26-33] and Polyakov linear sigma model (PLSM) $[34,35]$. However, the shortcoming of these models is that the temperature dependence of the Polyakov-loop potential is put in by hand from lattice result, which cannot be selfconsistently extended to finite baryon density. Recently, efforts have been made in Ref.[36] to derive a low-energy 
effective theory for confinement-deconfinement and chiralsymmetry breaking/restoration from first-principle.

Recent investigations revealed that quark propagator heat kernels can also act as an order parameter as they transform non trivially under the center transformation related to deconfinement transition [37-39]. But the exciting result is the behavior of spectral sum of the Dirac operator under center transformation [38,40-42]. A new order parameter, called dressed Polyakov loop has been defined which can be represented as a spectral sum of the Dirac operator [42]. It has been found the infrared part of the spectrum particularly plays a leading role in confinement [38]. This result is encouraging since it gives a hope to relate the chiral phase transition with the confinementdeconfinement phase transition. The order parameter for chiral phase transition is related to the spectral density of the Dirac operator through Banks-Casher relation [4]. Therefore, both the dressed Polyakov loop and the chiral condensate are related to the spectral sum of Dirac operator.

Behavior of the dressed Polyakov loop is mainly studied in the framework of Lattice gauge theory [43,44]. Apart from that, studies based on Dyson-Schwinger equations [45-47] and PNJL model [48] have been carried out. In those studies the role of dressed Polyakov loop as an order parameter is discussed at zero chemical potential. In this paper, we show our results [49] of investigating the QCD phase diagram at finite temperature and density by using the dressed Polyakov loop as an equivalent order parameter in the Nambu-Jona-Lasinio (NJL) model . It is known that he NJL model lacks of confinement and the gluon dynamics is encoded in a static coupling constant for four point contact interaction. However, assuming that we can read the information of confinement from the dual chiral condensate, it would be interesting to see the behavior of the dressed Polyakov loop in a scenario without any explicit mechanism for confinement.

In this paper, we show the phase transitions in the twoflavor and three-flavor NJL models in $(T, \mu)$ plane in chiral limit as well for small quark mass limit. This paper is organized as follows: We introduce the dressed Polyakov loop as an equivalent order parameter of confinement deconfinement phase transition and the NJL model in Sec. 2. Then in Sec.3, we show the results of two-flavor QCD phase diagram in $T-\mu$ plane in the chiral limit and in the case of explicit chiral symmetry breaking, respectively. We offer an analysis on the relation between the chiral and deconfinement phase transitions in Sec. 4. In Sec.5, we show the phase diagram at finite temperature and density for three-flavor case. At the end, we give the conclusion and discussion.

\section{Dressed Polyakov loop and the NJL model}

We firstly introduce the dressed Polyakov loop. To do this we have to consider a $U(1)$ valued boundary condition for the fermionic fields in the temporal direction instead of the canonical choice of anti-periodic boundary condition,

$$
\psi(x, \beta)=e^{-i \phi} \psi(x, 0),
$$

where $0 \leq \phi<2 \pi$ is the phase angle and $\beta$ is the inverse temperature.

Dual quark condensate $\Sigma_{n}$ is then defined by the Fourier transform (w.r.t the phase $\phi$ ) of the general boundary condition dependent quark condensate [42-44],

$$
\Sigma_{n}=-\int_{0}^{2 \pi} \frac{d \phi}{2 \pi} e^{-i n \phi}\langle\bar{\psi} \psi\rangle_{\phi}
$$

where $n$ is the winding number.

Particular case of $n=1$ is called the dressed Polyakov loop which transforms in the same way as the conventional thin Polyakov loop under the center symmetry and hence is an order parameter for the deconfinement transition [4244]. It reduces to the thin Polyakov loop and to the dual of the conventional chiral condensate in infinite and zero quark mass limits respectively, i.e., in the chiral limit $m \rightarrow$ 0 we get the dual of the conventional chiral condensate and in the $m \rightarrow \infty$ limit we have thin Polyakov loop [42-44].

The Lagrangian of three-flavor NJL model [50] is given as

$$
\begin{aligned}
\mathcal{L} & =\bar{\psi}\left(i \gamma^{\mu} \partial_{\mu}-m\right) \psi+G_{s} \sum_{a}\left\{\left(\bar{\psi} \tau_{a} \psi\right)^{2}+\left(\bar{\psi} i \gamma_{5} \tau_{a} \psi\right)^{2}\right\} \\
& -K\left\{\operatorname{Det}_{f}\left[\bar{\psi}\left(1+\gamma_{5}\right) \psi\right]+\operatorname{Det}_{f}\left[\bar{\psi}\left(1-\gamma_{5}\right) \psi\right]\right\}
\end{aligned}
$$

Where $\psi=(u, d, s)^{T}$ denotes the transpose of the quark field, and $m=\operatorname{Diag}\left(m_{u}, m_{d}, m_{s}\right)$ is the corresponding mass matrix in the flavor space. $\tau_{a}$ with $a=1, \cdots, N_{f}^{2}-1$ are the eight Gell-Mann matrices, and $\operatorname{Det}_{f}$ means determinant in flavor space. The last term is the standard form of the 't Hooft interaction, which is invariant under $S U(3)_{L} \times$ $S U(3)_{R} \times U(1)_{B}$ symmetry, but breaks down the $U_{A}(1)$ symmetry.

The $\phi$ dependent thermodynamic potential in the mean field level is given as following:

$\Omega_{\phi}=\sum_{f} \Omega_{\phi, M_{f}}+2 G_{s} \sum_{f}\langle\sigma\rangle_{\phi, f}^{2}-4 K\langle\sigma\rangle_{\phi, u}\langle\sigma\rangle_{\phi, d}\langle\sigma\rangle_{\phi, s}$,

with

$$
\begin{aligned}
\Omega_{\phi, M_{f}} & =-2 N_{c} \int_{\Lambda} \frac{d^{3} p}{(2 \pi)^{3}}\left[E_{p, f}+\frac{1}{\beta} \ln \left(1+e^{-\beta E_{p, f}^{-}}\right)\right. \\
& \left.+\frac{1}{\beta} \ln \left(1+e^{-\beta E_{p, f}^{+}}\right)\right] .
\end{aligned}
$$

Where the sum is in the flavor space, $E_{p, f}=\sqrt{p^{2}+M_{\phi, f}^{2}}$ and $E_{p, f}^{ \pm}=E_{p, f} \pm[\mu+i(\phi-\pi) T]$, with the constituent quark mass

$$
M_{\phi, i}=m_{i}-4 G_{s}\langle\sigma\rangle_{\phi, i}+2 K\langle\sigma\rangle_{\phi, j}\langle\sigma\rangle_{\phi, k},
$$

where $(i, j, k)$ is the quark flavor indices $(u, d, s)$, and $\langle\sigma\rangle_{\phi, f}=$ $\left\langle\bar{\psi}_{f} \psi_{f}\right\rangle_{\phi}$. 


\section{Phase diagram for two-flavor case}

We firstly show the results in the two-flavor case and consider the isospin symmetric limit, i.e, we take $N_{f}=2$, $K=0$ and $m_{u}=m_{d}$ in Eq.(3). The thermodynamic potential contains imaginary part. We take only the real part of the potential and the imaginary phase factor is not considered in this work. The mean field $\langle\sigma\rangle_{\phi}$ is obtained by minimizing the potential for each value of $\phi \in[0,2 \pi)$ for fixed $\mathrm{T}$ and $\mu$. The conventional chiral condensate is $\langle\sigma\rangle_{\pi}=\langle\bar{\psi} \psi\rangle_{\pi}$. The dressed Polyakov loop $\Sigma_{1}$ is obtained by integrating over the angle. The values of the parameters $\Lambda$ and $G_{s}$ are taken as $0.6315 \mathrm{GeV}$ and $5.498 \mathrm{GeV}^{-2}$, respectively.

We investigate phase transitions for two cases, i.e., in the chiral limit and with explicit chiral symmetry breaking. Fig. 1 and 2 show the behavior of the angle dependence of the general chiral condensate for various chemical potentials and temperatures for $m=0$ and $m=5.5 \mathrm{MeV}$, respectively. The four curves presented in each figure represent two temperatures above and below the critical temperature for two particular values of the chemical potential. Same qualitative features have been found for both the quark masses. The variation is symmetrical around $\phi=\pi$ as reported in other studies $[45,48]$.

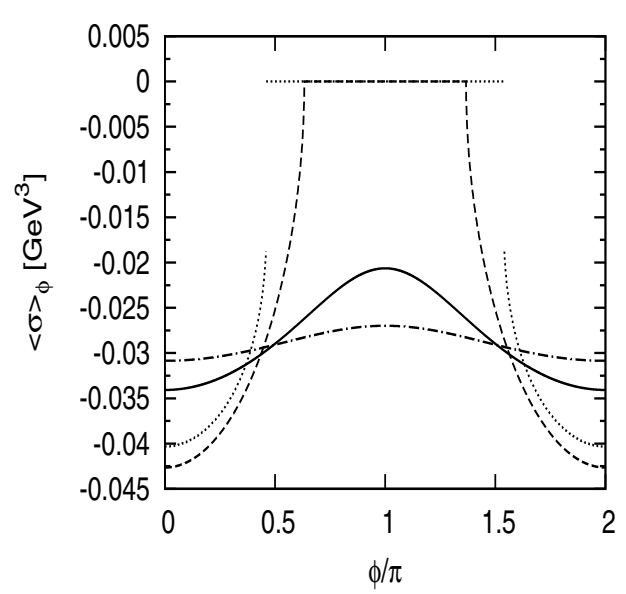

Fig. 1. Angle variation of $\langle\sigma\rangle_{\phi}$ for different values of temperatures and chemical potentials in the case of chiral limit. The solid line corresponds to $T=150 \mathrm{MeV}, \mu=100 \mathrm{MeV}$, dashed line corresponds to $T=250 \mathrm{MeV}, \mu=100 \mathrm{MeV}$, dash-dotted line corresponds to $T=40 \mathrm{MeV}, \mu=300 \mathrm{MeV}$, and dot corresponds to $T=150 \mathrm{MeV}, \mu=300 \mathrm{MeV}$.

Almost no variation with respect to angle is found for low temperatures. As the temperature increases the variation over the angle grows. We expect the absolute value of the chiral condensate decreases with the increase of temperature. However, from the figure, this conventional behavior of the chiral condensate with temperature only persists up to a certain angle, beyond which the opposite behavior is observed. The plateau around $\phi=\pi$ is more flat above $T_{c}$ in case of zero current quark mass and its value

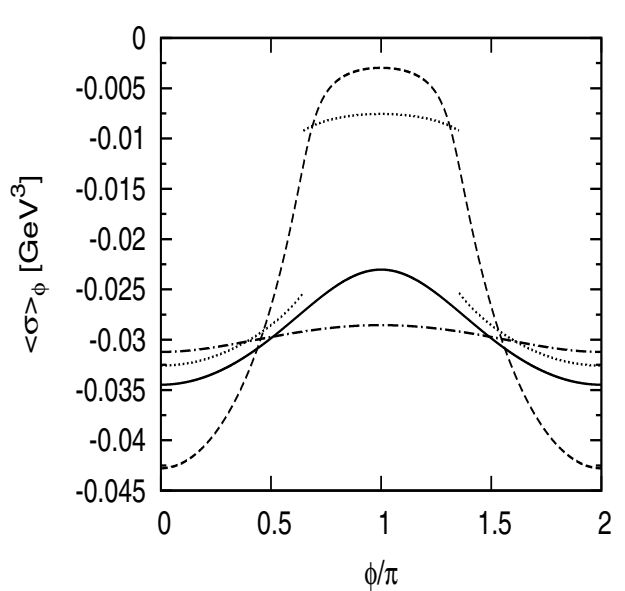

Fig. 2. Angle variation of $\langle\sigma\rangle_{\phi}$ for different values of temperatures and chemical potentials in the case of $m=5.5 \mathrm{MeV}$. The solid line corresponds to $T=150 \mathrm{MeV}, \mu=100 \mathrm{MeV}$, dashed line corresponds to $T=250 \mathrm{MeV}, \mu=100 \mathrm{MeV}$, dash-dotted line corresponds to $T=20 \mathrm{MeV}, \mu=340 \mathrm{MeV}$ and dotted line corresponds to $T=40 \mathrm{MeV}, \mu=340 \mathrm{MeV}$.

is consistent with the expectation of complete restoration of chiral symmetry in the chiral limit.

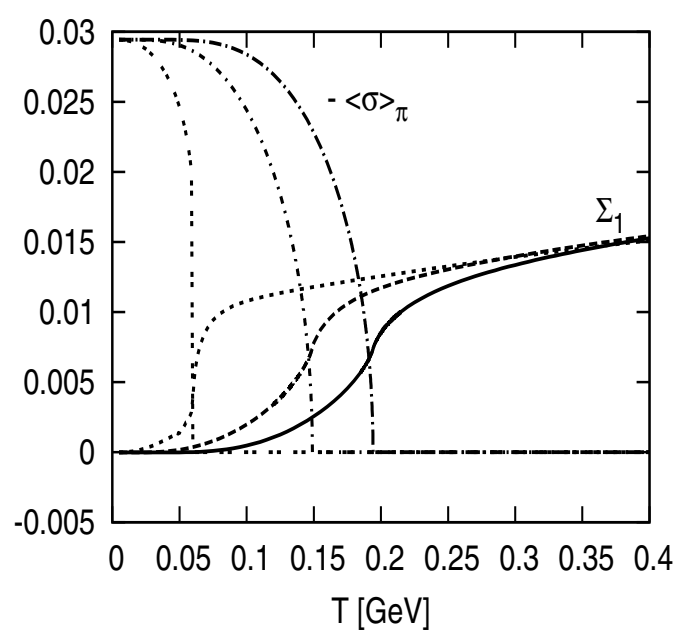

Fig. 3. The conventional chiral condensate $-\langle\sigma\rangle_{\pi}$ and the dressed Polyakov loop $\Sigma_{1}$ as functions of temperature for different values of the chemical potentials in the chiral limit. Here, $-\langle\sigma\rangle_{\pi}$ and $\Sigma_{1}$ both are measured in $\left[\mathrm{GeV}^{3}\right]$. From right to left the values of the chemical potential are $0,200,300 \mathrm{MeV}$, respectively.

Fig. 3 and 4 show the behavior of the conventional chiral condensate $-\langle\sigma\rangle_{\pi}$ and the dressed Polyakov loop $\Sigma_{1}$ at different chemical potentials as functions of temperature for $m=0$ and $m=5.5 \mathrm{MeV}$, respectively. For both cases, it is observed there are three temperature regions for $-\langle\sigma\rangle_{\pi}$ and $\Sigma_{1}$. For $-\langle\sigma\rangle_{\pi}$, at smaller temperatures it remains constant at a value corresponding to the value of the conventional chiral condensate in the vacuum, then it rapidly decreases in a small window of temperature and eventually almost saturates to a lower value. The rapid de- 


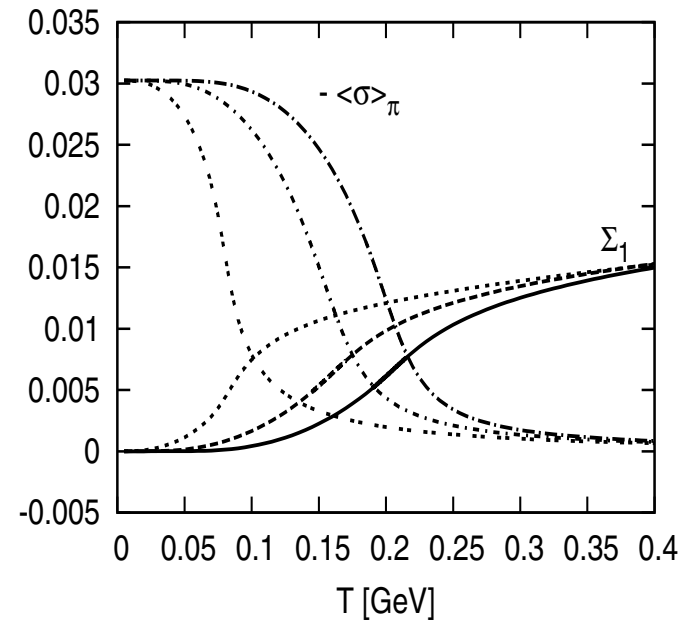

Fig. 4. The conventional chiral condensate $-\langle\sigma\rangle_{\pi}$ and the dressed Polyakov loop $\Sigma_{1}$ as functions of temperature for different values of the chemical potentials in the case of explicit chiral symmetry breaking $m=5.5 \mathrm{MeV}$ and $-\langle\sigma\rangle_{\pi}, \Sigma_{1}$ are measured in $\left[\mathrm{GeV}^{3}\right]$. From right to left the values of the chemical potential are $0,200,300 \mathrm{MeV}$, respectively.

creasing occurs at different temperatures for different values of the chemical potentials. On the other hand the behavior for the dressed Polyakov loop is just the opposite. It remains almost zero for small temperatures and then rises rapidly, finally saturates to a high value which varies very slowly with temperatures. The almost zero value of $\Sigma_{1}$ for small temperatures is due to the fact that the $U(1)$ boundary condition dependent general quark condensate nearly does not vary with the angle $\phi$ for small temperatures (see Eq. 2).

For finite quark mass, near the critical temperature region, both $-\langle\sigma\rangle_{\pi}$ and $\Sigma_{1}$ change more slowly than those in the case of chiral limit.

Fig. 5 and 6 show the $T-\mu$ phase diagram for the case of $m=0$ and $m=5.5 \mathrm{MeV}$, respectively. The transition temperatures are calculated from the slope analysis of the conventional chiral condensate $\langle\sigma\rangle_{\pi}$ and the dressed Polyakov loop. The transition temperatures calculated from the conventional chiral condensate represent chiral phase transition temperature. On the other hand the behavior of the dressed Polyakov loop is supposed to indicate the deconfinement transition temperature. In our present framework confinement is not accounted for. However, if we look at the curves presented in figure 3 and 4, they still show an order parameter like behavior. We would like to point out here that for the same reason as stated above we are not concerned here with the order of the phase transtion from the dressed Polyakov loop and all the comments about the order of the phase transition below are with respect to the chiral phase transition only.

For $m=0$ case, we find almost exact matching for the transition temperatures calculated from these two quantities in the whole $T-\mu$ plane as shown in Fig. 5.

For the case of finite quark mass $m=5.5 \mathrm{MeV}$, it is observed from Fig. 6 that the two critical temperatures are

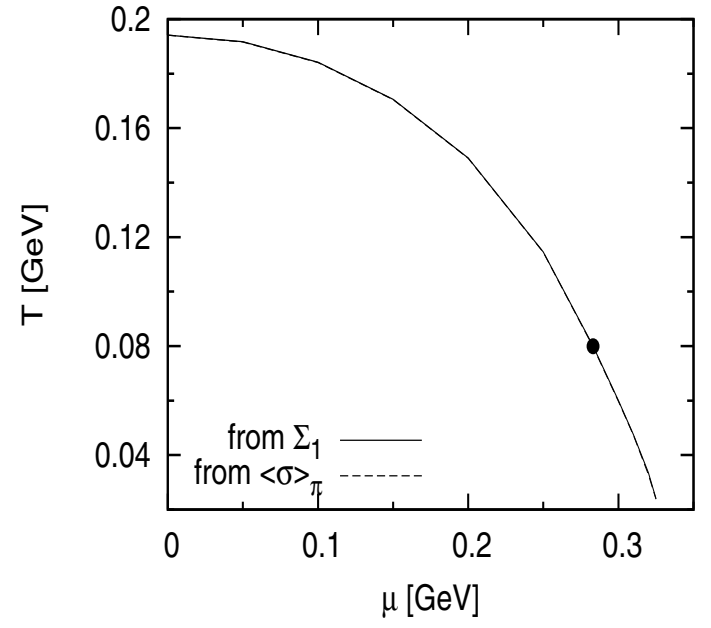

Fig. 5. Two-flavor phase diagram in the $T-\mu$ plane for the case of chiral limit. The solid line is the critical line for $\Sigma_{1}$, and the dashed line is the critical line for conventional chiral phase transition. The solid circle indicates the critical point for chiral phase transition.

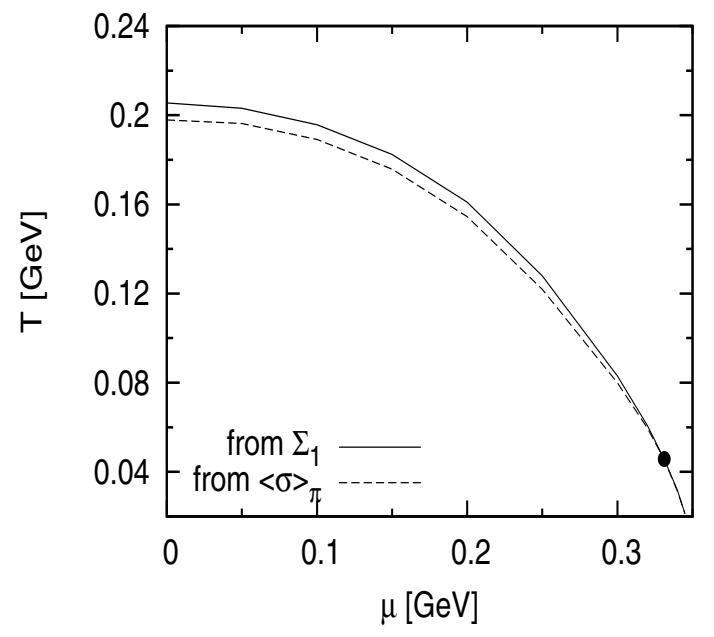

Fig. 6. Two-flavor phase diagram in the $T-\mu$ plane for the case of $m=5.5 \mathrm{MeV}$. The solid line is the critical line for $\Sigma_{1}$, and the dashed line is the critical line for conventional chiral phase transition. The solid circle indicates the critical end point for chiral phase transition.

different in the low baryon density region. The difference however decreases from low to high chemical potential, and the two critical temperatures start to match around the critical end point for chiral phase transition. For zero chemical potential and small current quark mass $m=5.5$ $\mathrm{MeV}$, we find about $7 \mathrm{MeV}$ difference between $T_{c}^{\chi}$ and $T_{c}^{\mathcal{D}}$, and $T_{c}^{\chi}<T_{c}^{\mathcal{D}}$. Similar trend has been observed in another study based on Dyson-Schwinger approach [45], where they found chiral transition to occur about $10-20 \mathrm{MeV}$ below the deconfinement transition. Though these studies are not a complete one and these differences may be due to the effects of crossover transition. As pointed out in [25] during crossover, different observables are expected to behave 
differently and there is no way to define a unique crossover temperature.

We extend our study further to see what happens if we increase the current quark mass further. We find at zero chemical potential, the difference between the transition temperatures calculated from dressed Polyakov loop and conventional chiral condensate increases as we increase the current quark mass (see Fig. 7). Initially the difference is zero for zero current quark mass but for $m=200 \mathrm{MeV}$ we find about $26 \mathrm{MeV}$ difference between the two temperatures. It is worthy of mentioning that this result is just for illustrative purpose as there are limitation of using NJL model with such a huge current quark mass.

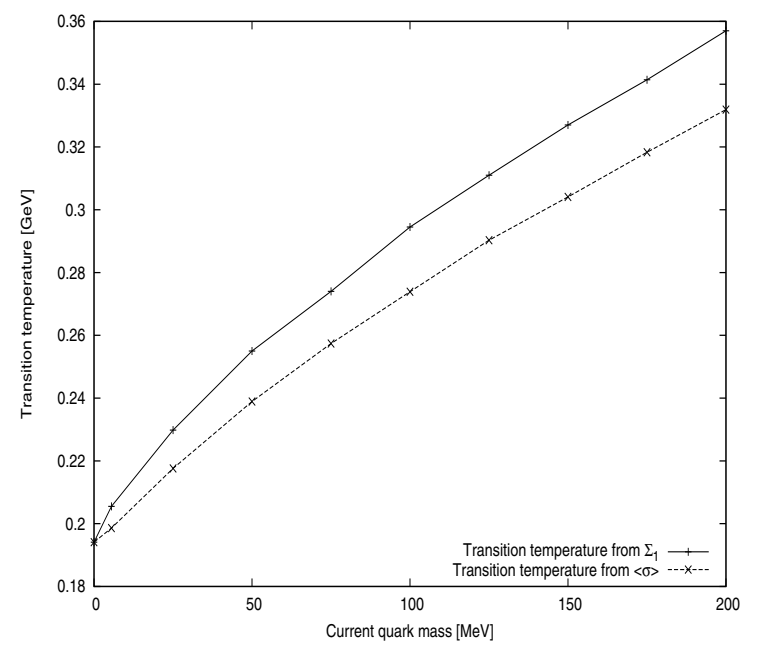

Fig. 7. The critical temperatures $T_{c}^{\chi}$ and $T_{c}^{\mathcal{D}}$ for different current masses.

\section{The relation between $T_{c}^{\chi}$ and $T_{c}^{\mathcal{D}}$}

In the following, we offer a possible understanding on the simultaneity of the transition temperatures for 1 st and 2 nd order chiral phase transitions and the apparent difference between the two for the case of crossover.

As mentioned earlier the transition temperatures are determined from the slope analysis of the conventional chiral condensate and the dressed Polyakov loop. So let us look at the temperature derivative of the general chiral condensate $d\langle\sigma\rangle_{\phi} / d T$ as functions of $\phi$, the integral on which gives the temperature derivative of the Dressed Polyakov loop

$$
\frac{d \Sigma_{1}}{d T}=-\int_{0}^{2 \pi} \frac{d \phi}{2 \pi} e^{-i \phi} \frac{d\langle\sigma\rangle_{\phi}}{d T} .
$$

Fig. 8 shows $d\langle\sigma\rangle_{\phi} / d T$ at different temperatures in the cases of second order chiral phase transitions. Around $T_{c}^{\chi}$, large values of $d\langle\sigma\rangle_{\phi} / d T$ appear around $\phi=\pi$ and dominate the integral in Eq. (7). Below $T_{c}^{\chi}, d\langle\sigma\rangle_{\phi} / d T$ around $\phi=\pi$ increases monotonously as temperature increases. As a result, $d \Sigma_{1} / d T$ increases. Above $T_{c}^{\chi}, d\langle\sigma\rangle_{\phi} / d T$ in the center region becomes zero (or very small in the case with

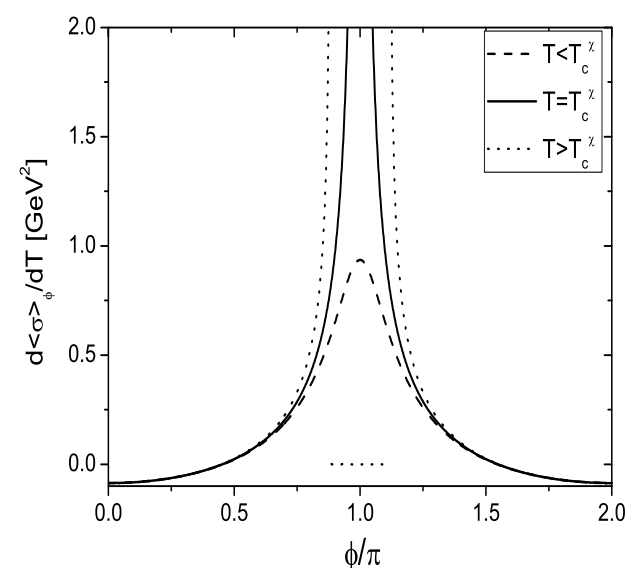

Fig. 8. Temperature derivative of the general chiral condensate $\frac{d\langle\sigma\rangle_{\phi}}{d T}$ for $m=0$ and $\mu=0$ at three temperature cases: $T<T_{c}{ }^{\chi}$, $T=T_{c}{ }^{\chi}$ and $T>T_{c}{ }^{\chi}$, where $T_{c}{ }^{\chi}$ is the chiral transition temperature.

finite current quark mass) and the region with large values of $d\langle\sigma\rangle_{\phi} / d T$ shrinks. Therefore, the integral in Eq. (7) i.e. $d \Sigma_{1} / d T$ decreases as temperature increases. In all, $d \Sigma_{1} / d T$ gets its maximum at $T_{c}^{\chi}$ and the two transition temperatures $T_{c}^{\mathcal{D}}$ and $T_{c}^{\chi}$ coincide in the case of second order chiral phase transition.

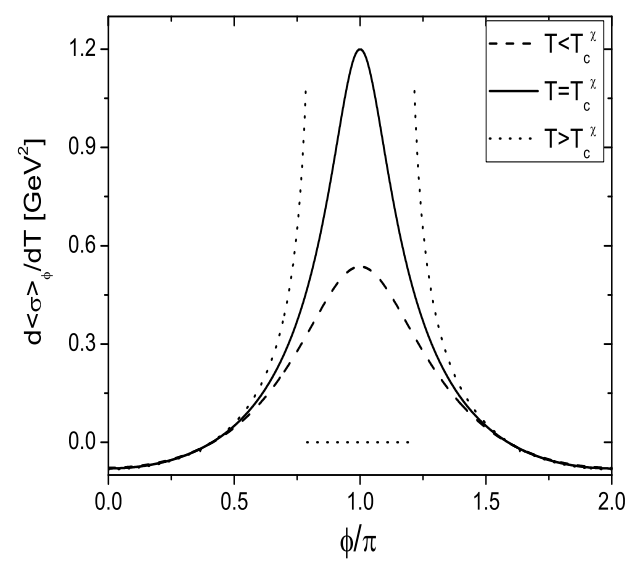

Fig. 9. Temperature derivative of the general chiral condensate $\frac{d\langle\sigma\rangle_{\phi}}{d T}$ for $m=0$ and $\mu=300 \mathrm{MeV}$ at three temperature cases: $T^{d T}<T_{c}{ }^{\chi}, T=T_{c}{ }^{\chi}$ and $T>T_{c}{ }^{\chi}$, where $T_{c}{ }^{\chi}$ is the chiral transition temperature.

In the case of first order chiral phase transition, the situation is more complicated. Due to the discontinuity of $\langle\sigma\rangle_{\phi}$ at the first order chiral phase transition point, the temperature derivative of the dressed Polyakov loop can be expressed as

$$
\frac{d \Sigma_{1}}{d T}=-\int_{0}^{2 \pi} \frac{d \phi}{2 \pi} e^{-i \phi} \frac{d\langle\sigma\rangle_{\phi}}{d T}-\frac{\operatorname{Cos} \phi_{c}}{\pi} \Delta\langle\sigma\rangle_{c} \frac{d \phi_{c}}{d T},
$$


where, the first term is determined by the regular behavior of $d\langle\sigma\rangle_{\phi} / d T$ (see Fig.9), the second term is due to $\Delta\langle\sigma\rangle_{c}$, the jump of $\langle\sigma\rangle_{\phi}$ at the first order phase transition point at $\phi=\phi_{c}$. When $T<T_{c}^{\chi}$, the second term vanishes. Now we consider two limiting cases. First, in the case of a weak first order phase transition, $\Delta\langle\sigma\rangle_{c}$ is small and $d\langle\sigma\rangle_{\phi} / d T$ around $\phi=\pi$ is large, as showed in Fig.9. So the first term dominates the result of Eq. (8) and gives the similar result as that in the case of a second order chiral phase transition. Second, in the case of a strong first order chiral phase transition, $\Delta\langle\sigma\rangle_{c}$ is large and $d\langle\sigma\rangle_{\phi} / d T$ is small. So the second term dominates the result of Eq. (8). Then $\frac{d \Sigma_{1}}{d T}$ is strongly dependent on the detailed information of $d \phi_{c} / d T$. Our numerical results show that $d \phi_{c} / d T$ decreases as temperature increases, and so the second term also gives a decreasing contribution. In all, it is clear that $d \Sigma_{1} / d T$ gets its maximum at $T_{c}^{\chi}$, i.e. $T_{c}^{\mathcal{D}}$ and $T_{c}^{\chi}$ coincide in the case of a weak first order chiral phase transition due to remnants of second order chiral phase transition. The coincidence in the case of a general first order chiral phase transition is supported by our numerical results and can be generally expected.

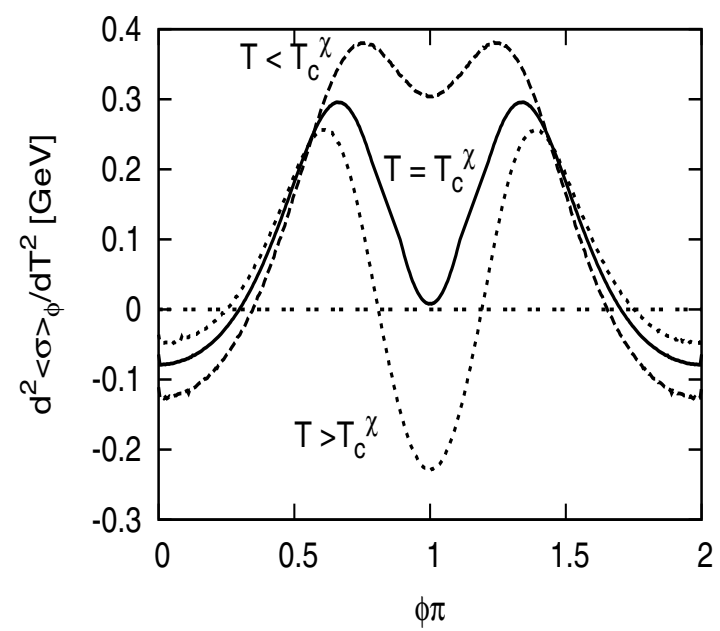

Fig. 10. Second derivative of the general chiral condensate $\frac{d^{2}\langle\sigma\rangle_{\phi}}{d T^{2}}$ for $m=5.5 \mathrm{MeV}$ and $\mu=0$ at three temperature cases: $\stackrel{d T^{2}}{T}<$ $T_{c}{ }^{\chi}, T=T_{c}{ }^{\chi}$ and $T>T_{c}{ }^{\chi}$, where $T_{c}{ }^{\chi}$ is the chiral transition temperature.

For the case with finite quark mass and small chemical potential, we have no phase transitions but crossover. So let us consider the second temperature derivative of the dressed Polyakov loop

$$
\frac{d^{2} \Sigma_{1}}{d T^{2}}=-\int_{0}^{2 \pi} \frac{d \phi}{2 \pi} e^{-i \phi} \frac{d^{2}\langle\sigma\rangle_{\phi}}{d T^{2}}
$$

whose zero point determines the transition temperature $T_{c}^{\mathcal{D}}$. Fig. 10 shows the second derivatives of the general chiral condensate $d^{2}\langle\sigma\rangle_{\phi} / d T^{2}$ at three temperature cases: $T<$ $T_{c}{ }^{\chi}, T=T_{c}{ }^{\chi}$ and $T>T_{c}{ }^{\chi}$, where $T_{c}{ }^{\chi}$ is the chiral transition temperature. Similar to our previous observation, large values of $d^{2}\langle\sigma\rangle_{\phi} / d T^{2}$ appear around $\phi=\pi$ (see the two maximums in Fig. 10), as remnants of the second order phase transition in chiral limit. The difference is that, the $d^{2}\langle\sigma\rangle_{\phi} / d T^{2}$ in the center region (see the minimum in Fig. 10), is suppressed below $T_{c}{ }^{\chi}$, approaches zero at $T_{c}{ }^{\chi}$ and changes its sign above $T_{c}{ }^{\chi}$. For $T \leq T_{c}{ }^{\chi}$, the $d^{2}\langle\sigma\rangle_{\phi} / d T^{2}$ around the two maximums dominate the integral in Eq.(7) and $d^{2} \Sigma_{1} / d T^{2}$ does not change its sign. Above $T_{c}{ }^{x}$, the negative part around the minimum cancels the contributions from the maximums, and up to a certain temperature $T_{c}^{\mathcal{D}}$, this cancelation leads to the zero of the integral in Eq.(9). In all, the zero point of $d^{2} \Sigma_{1} / d T^{2}$ comes from the negative contribution of the minimum at $T>T_{c}{ }^{\chi}$, so the transition temperature related with the dressed Polyakov loop must be higher than the chiral transition temperature, i.e. $T_{c}{ }^{\mathcal{D}}>T_{c}{ }^{\chi}$.

\section{Phase diagram for three-flavor case}

We now consider the phase diagram for three-flavor case, and the parameters are taken from Ref.[51,52]:

\begin{tabular}{|c|c|c|c|c|}
\hline & $m_{q}[\mathrm{MeV}]$ & $m_{s}[\mathrm{MeV}]$ & $G_{s} \Lambda^{2}$ & $K \Lambda^{5}$ \\
\hline Chiral-limit & 0 & 0 & 1.926 & 12.36 \\
\hline finite-mass & 5.5 & 140.7 & 1.918 & 12.36 \\
\hline
\end{tabular}

Table 1. Two sets of parameters in 3-flavor NJL model: the current quark mass $m_{q}$ for up and down quark and $m_{s}$ for strange quark, coupling constants $G$ and $K$, with a spatial momentum cutoff $\Lambda=602.3 \mathrm{MeV}$.

In the chiral limit, i.e, for the case of $m_{u}=m_{d}=m_{s}=$ 0 case, we find almost exact matching for the transition temperatures calculated from these two quantities in the whole $T-\mu$ plane as shown in Fig. 11. The chiral phase transition is of 1 st order in the whole $T-\mu$ plane.

For the case of finite quark mass $m_{u}=m_{d}=5.5 \mathrm{MeV}$ and $m_{s}=140.7 \mathrm{MeV}$, it is observed from Fig. 12 that the the chiral and deconfinement phase transitions are flavor dependent. For the degenerate light $u, d$ quarks, the result of phase transitions are similar to that in Fig. 6. The critical temperatures of chiral and deconfinement phase transitions have very small difference in the low baryon density region when the transition is of crossover, and the difference vanishes at the critical end point. Here the CEP is located at $\left(T_{C E P}^{u, d}, \mu_{C E P}^{u, d}\right)=(91 \mathrm{MeV}, 315 \mathrm{MeV})$, which is different from Fig. 6. This difference comes from: 1) different model parameters have been used, 2) the coupling of $s$ quark to $u, d$ quark contributes one extra term in the thermodynamical potential comparing with the pure two-flavor case. For the relative heavy $s$ quark, it is found that this quark has separate phase transition lines. At low baryon density, the variation of s quark condensate in the crossover region is so diffused that it is not possible to identify the crossover temperature. When density increases, the crossover temperatures for chiral and deconfinement can be effectively extracted and still shows the relation of $T_{c}^{\chi}<T_{c}^{\mathcal{D}}$. These two critical temperatures of $s$ quark coincide at the critical end point $\left(T_{C E P}^{s}, \mu_{C E P}^{s}\right)=(75 \mathrm{MeV}, 445 \mathrm{MeV})$. 


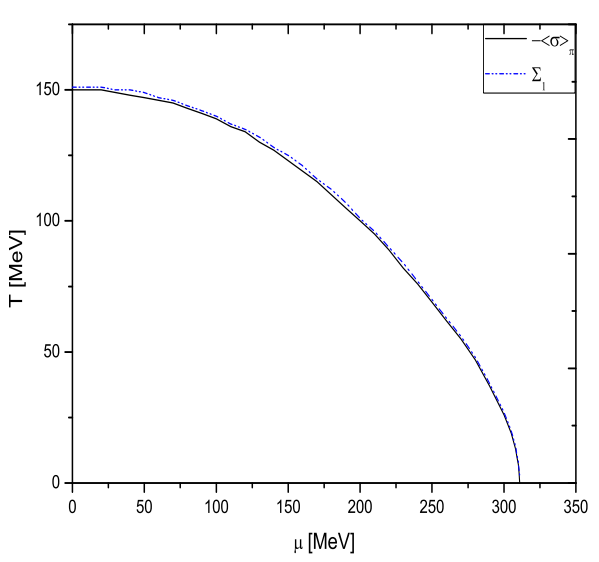

Fig. 11. Three-flavor phase diagram in the $T-\mu$ plane for the case of chiral limit. The solid line is the critical line for $\Sigma_{1}$, and the dashed line is the critical line for conventional chiral phase transition.

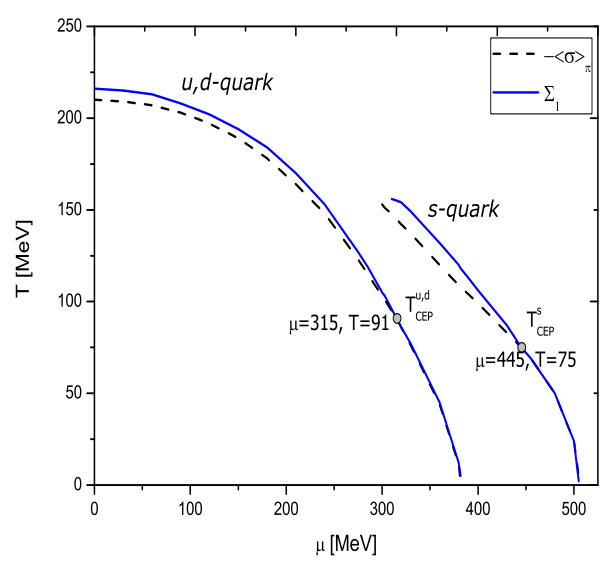

Fig. 12. Three-flavor phase diagram in the $T-\mu$ plane for the case of $m_{u}=m_{d}=5 \mathrm{MeV}$ and $m_{s}=140.7 \mathrm{MeV}$. The solid lines are the critical line for $\Sigma_{1}$, and the dashed lines are the critical line for conventional chiral phase transition. The solid circles indicate the critical end points for chiral phase transitions of $u, d$ and $s$ quark, respectively.

\section{Conclusion and discussion}

We investigate the chiral condensate and the dressed Polyakov loop or dual chiral condensate at finite temperature and density in the two and three-flavor Nambu-Jona-Lasinio model. We find the behavior of dressed Polyakov loop in absence of any confinement mechanism still shows an order parameter like behavior. It is found that in the chiral limit, the critical temperature for chiral phase transition coincides with that of the dressed Polyakov loop. In the case of explicit chiral symmetry breaking, it is found that the phase transitions are flavor dependent, and the critical temperature for chiral transition $T_{c}^{\chi}$ is smaller than that of the dressed Polyakov loop $T_{c}^{\mathcal{D}}$ in the low baryon density re- gion where the transition is a crossover. With the increase of current quark mass the difference between the two critical temperatures is found to be increasing. However, the two critical temperatures coincide in the high baryon density region where the phase transition is of first order. For three-flavor case, it is observed that there are two critical end points in the $(T, \mu)$ phase diagram.

From symmetry analysis, the dressed Polyakov loop can be regarded as an equivalent order parameter of deconfinement phase transition for confining theory. In the NJL model, the gluon dynamics is encoded in a static coupling constant for four point contact interaction. Since in this work we have included only quark degrees of freedom, a quantitative comparison will not match with other results. But the interesting fact is that the qualitative features (angle variation, temperature variation) of the dressed Polyakov loop remains the same. Moreover, we expect that independent of the input of gluedynamics to the quark propagator, it is a general feature for $T_{c}^{\chi}=T_{c}^{\mathcal{D}}$ in the case of 1st and 2 nd order phase transitions, and $T_{c}^{\chi}<T_{c}^{\mathcal{D}}$ in the case of crossover, which qualitatively agrees with the lattice result in Ref. [25]. This might indicate that for full QCD, in the crossover case, there exists a small region where chiral symmetry is restored but the color degrees of freedom are still confined. This result should be checked in other effective models, e.g. in the framework of Dyson-Schwinger equations (DSE).

The $(T, \mu)$ phase diagram with three flavors and with $U_{A}(1)$ anomaly as well as diquark condensate will be studied in the near future.

Acknowledgments: M.H. thanks P. Levai and local organizers for hospitality, and she also acknowledges Hungarian Academy of Sciences for supporting the local expenses. The work of M.H. is supported by CAS program "Outstanding young scientists abroad brought-in", and the NSFC under the number of 10735040 and 10875134 , and K.C.Wong Education Foundation, Hong Kong.

\section{References}

1. A. M. Polyakov, Phys. Lett. B 72, 477 (1978).

2. G. 't Hooft, Nucl. Phys. B 138, 1 (1978).

3. A. Casher, Phys. Lett. B 83, 395 (1979).

4. T. Banks and A. Casher, Nucl. Phys. B 169, 103 (1980).

5. Y. Hatta and K. Fukushima, Phys. Rev. D 69, 097502 (2004)

6. A. Mocsy, F. Sannino and K. Tuominen, Phys. Rev. Lett. 92, 182302 (2004).

7. F. Marhauser and J. M. Pawlowski, arXiv:0812.1144 [hep-ph].

8. J. Braun, H. Gies and J. M. Pawlowski, Phys. Lett. B 684, 262 (2010).

9. J. Braun, L. M. Haas, F. Marhauser and J. M. Pawlowski, arXiv:0908.0008 [hep-ph].

10. T. Hatsuda and K. Maeda, arXiv:0912.1437 [hepph]; K. Fukushima and T. Hatsuda, Rept. Prog. Phys. 
74, 014001 (2011); J. M. Pawlowski, arXiv:1012.5075 [hep-ph].

11. L. McLerran and R. D. Pisarski, Nucl. Phys. A 796, 83 (2007)

12. K. Rajagopal and F. Wilczek, hep-ph/0011333; D.K. Hong, Acta Phys. Polon. B 32, 1253 (2001); M. Alford, Ann. Rev. Nucl. Part. Sci. 51, 131 (2001); G. Nardulli, Riv. Nuovo Cim. 25N3, 1 (2002); T. Schäfer, hep-ph/0304281; H.C. Ren, hepph/0404074; M. Huang, Int. J. Mod. Phys. E 14, 675 (2005); I. A. Shovkovy, Found. Phys. 35, 1309 (2005); M. G. Alford, A. Schmitt, K. Rajagopal and T. Schafer, Rev. Mod. Phys. 80, 1455 (2008); Q. Wang, arXiv:0912.2485 [nucl-th];

13. M. Panero, Phys. Rev. Lett. 103, 232001 (2009).

14. J. B. Kogut, M. Stone, H. W. Wyld, W. R. Gibbs, J. Shigemitsu, S. H. Shenker and D. K. Sinclair, Phys. Rev. Lett. 50, 393 (1983).

15. M. Fukugita and A. Ukawa, Phys. Rev. Lett. 57, 503 (1986).

16. F. Karsch and E. Laermann, Phys. Rev. D 50, 6954 (1994).

17. S. Digal, E. Laermann and H. Satz, Eur. Phys. J. C 18, 583 (2001).

18. S. Digal, E. Laermann and H. Satz, Nucl. Phys. A 702, 159 (2002).

19. F. Karsch, Lect. Notes Phys. 583, 209 (2002).

20. E. Laermann and O. Philipsen, Ann. Rev. Nucl. Part. Sci. 53, 163 (2003).

21. C. Bernard et al. [MILC Collaboration], Phys. Rev. D 71, 034504 (2005).

22. M. Cheng et al., Phys. Rev. D 74, 054507 (2006).

23. Y. Aoki, Z. Fodor, S. D. Katz and K. K. Szabo, Phys. Lett. B 643, 46 (2006).

24. Y. Aoki, S. Borsanyi, S. Durr, Z. Fodor, S. D. Katz, S. Krieg and K. K. Szabo, JHEP 0906, 088 (2009).

25. Z. Fodor and S. D. Katz, arXiv:0908.3341 [hep-ph].

26. K. Fukushima, Phys. Lett. B 591, 277 (2004).

27. C. Ratti, M. A. Thaler and W. Weise, Phys. Rev. D 73, 014019 (2006).

28. C. Sasaki, B. Friman and K. Redlich, Phys. Rev. D 75, 074013 (2007) [arXiv:hep-ph/0611147].

29. S. K. Ghosh, T. K. Mukherjee, M. G. Mustafa and R. Ray, Phys. Rev. D 73, 114007 (2006).

30. W. j. Fu, Z. Zhang and Y. x. Liu, Phys. Rev. D 77, 014006 (2008).

31. Z. Zhang and Y. X. Liu, Phys. Rev. C 75, 064910 (2007).

32. K. Fukushima, Phys. Rev. D 77, 114028 (2008) [Erratum-ibid. D 78, 039902 (2008)].

33. H. Abuki, R. Anglani, R. Gatto, G. Nardulli and M. Ruggieri, Phys. Rev. D 78, 034034 (2008).

34. B. J. Schaefer, J. M. Pawlowski and J. Wambach, Phys. Rev. D 76, 074023 (2007).

35. H. Mao, J. Jin and M. Huang, J. Phys. G 37, 035001 (2010).

36. K. I. Kondo, arXiv:1005.0314 [hep-th].

37. F. Synatschke, A. Wipf and C. Wozar, Phys. Rev. D 75, 114003 (2007).
38. F. Synatschke, A. Wipf and K. Langfeld, Phys. Rev. D 77, 114018 (2008).

39. E. Bilgici and C. Gattringer, JHEP 0805, 030 (2008).

40. C. Gattringer, Phys. Rev. Lett. 97, 032003 (2006).

41. F. Bruckmann, C. Gattringer and C. Hagen, Phys. Lett. B 647, 56 (2007).

42. E. Bilgici, F. Bruckmann, C. Gattringer and C. Hagen, Phys. Rev. D 77, 094007 (2008).

43. F. Bruckmann, C. Hagen, E. Bilgici and C. Gattringer, PoS CONFINEMENT8, 054 (2008).

44. E. Bilgici, $\mathrm{PhD}$ Thesis, University of Garz, Austria, $2009 \quad$ (http://physik.unigarz.at/itp/files/bilgici/dissertation.pdf).

45. C. S. Fischer, Phys. Rev. Lett. 103, 052003 (2009).

46. C. S. Fischer and J. A. Mueller, Phys. Rev. D 80, 074029 (2009).

47. C. S. Fischer, A. Maas and J. A. Mueller, arXiv:1003.1960 [hep-ph].

48. K. Kashiwa, H. Kouno and M. Yahiro, Phys. Rev. D 80, 117901 (2009).

49. T. K. Mukherjee, H. Chen and M. Huang, Phys. Rev. D 82, 034015 (2010); F. K. Xu, T. K. Mukherjee, M. Huang, in preparation.

50. U. Vogl and W. Weise, Prog. Part. Nucl. Phys. 27, 195 (1991); S.P. Klevansky, Rev. Mod. Phys. 64, 649 (1992); T. Hatsuda and T. Kunihiro, Phys. Rept. 247, 221 (1994); R. Alkofer, H. Reinhardt and H. Weigel, Phys. Rept. 265, 139 (1996);

51. M. Buballa, Phys. Rept. 407, 205 (2005).

52. H. Abuki, G. Baym, T. Hatsuda and N. Yamamoto, Phys. Rev. D 81, 125010 (2010). 